\title{
Delivery by Destructive Operation
}

National Cancer Institute

\section{Source}

National Cancer Institute. Delivery by Destructive Operation. NCI Thesaurus. Code C114143.

Extraction of a fetus through the cervix using destructive surgical techniques. 